University of Wollongong

Research Online

Faculty of Informatics - Papers (Archive)

Faculty of Engineering and Information

Sciences

June 2004

\title{
Multi-modulation schemes to increase the rate space-time block codes in Rayleigh fading channels
}

\author{
Le Chung Tran \\ University of Wollongong, Ictran@uow.edu.au \\ Tadeusz A. Wysocki \\ University of Wollongong, wysocki@uow.edu.au \\ Alfred Mertins \\ University of Oldenburg, Germany, mertins@uow.edu.au
}

Follow this and additional works at: https://ro.uow.edu.au/infopapers

Part of the Physical Sciences and Mathematics Commons

\section{Recommended Citation}

Tran, Le Chung; Wysocki, Tadeusz A.; and Mertins, Alfred: Multi-modulation schemes to increase the rate space-time block codes in Rayleigh fading channels 2004.

https://ro.uow.edu.au/infopapers/110

Research Online is the open access institutional repository for the University of Wollongong. For further information contact the UOW Library: research-pubs@uow.edu.au 


\title{
Multi-modulation schemes to increase the rate space-time block codes in Rayleigh fading channels
}

\author{
Abstract \\ The paper examines multimodulation schemes (MMSs) to increase the rate of our two new complex \\ orthogonal designs (CODs) proposed for eight transmit antennas, namely C/sub $1 /$ and C/sub $2 /$, \\ corresponding to the Amicable Orthogonal Designs (AODs) $(8 ; 1,1,2,2 ; 1,1,2,2)$ and $(8 ; 1,1,1,4 ; 1,1,1,4)$, \\ respectively. In addition, the optimal intersymbol power allocation in the proposed codes in single \\ modulation as well as in MMSs in flat Rayleigh fading channels is considered. It turns out that, in some \\ modulation schemes, equal power transmission per each symbol time slot is not only optimal from the \\ technical point of view, but also optimal in terms of achieving the best symbol error probability. The \\ principles examining the MMSs in order to increase the rate of space-time block codes (STBCs) and the \\ optimal power allocation for multimodulated STBCs mentioned here can be generalized for STBCs of \\ other orders without any difficulty.

\section{Keywords} \\ Rayleigh channels, antennas, block codes, modulation, space-time codes

\section{Disciplines} \\ Physical Sciences and Mathematics

\section{Publication Details} \\ This paper originally appeared as: Tran, LC, Wysocki, TA \& Mertins, A, Multi-modulation schemes to \\ increase the rate space-time block codes in Rayleigh fading channels, 2nd International Conference on \\ Information Technology: Research and Education, 28 June-1 July 2004, 19-23. Copyright IEEE 2004.
}




\section{Multi-modulation Schemes to Increase the Rate of Space-Time Block Codes in Rayleigh Fading Channels}

\author{
Le Chung Tran, Tadeusz A. Wysocki, Jennifer Seberry \\ University of Wollongong \\ Northfields Avenue, NSW 2522, Australia \\ Email: lct71,wysocki,jennie@uow.edu.au
}

\author{
Alfred Mertins \\ University of Oldenburg \\ 26111 Oldenburg, Germany \\ Email: alfred.mertins@uni-oldenburg.de
}

\begin{abstract}
The paper examines multi-modulation schemes (MMSs) to increase the rate of our two new Complex Orthogonal Designs (CODs) proposed for eight transmit antennas, namely $C_{1}$ and $C_{2}$, corresponding to the Amicable Orthogonal Designs (AODs) $(8 ; 1,1,2,2 ; 1,1,2,2)$ and $(8 ; 1,1,1,4 ; 1,1,1,4)$, respectively. In addition, the optimal inter-symbol power allocation in the proposed codes in single modulation as well as in MMSs in flat Rayleigh fading channels is considered. It turns out that, in some modulation schemes, equal power transmission per each symbol time slot is not only optimal from the technical point of view, but also optimal in terms of achieving the best symbol error probability. The principles examining the MMSs in order to increase the rate of space-time block codes (STBCs) and the optimal power allocation for multi-modulated STBCs mentioned here can be generalized for STBCs of other orders without any difficulty.
\end{abstract}

\section{INTRODUCTION}

It is well known that STBCs of complex signals for more than two transmit antennas with full diversity cannot provide a full rate. The maximum rates of those STBCs for 4 and 8 transmit antennas are $3 / 4$ and $1 / 2$, respectively [1]. The STBCs for more than two transmit antennas are attractive in the sense that they provide more spatial diversity and temporal diversity for transmitted symbols, and consequently, provide better bit error performance than those for two transmit antennas. Additionally, they provide full spatial diversity for a given number of transmit antennas. To date, the existing STBCs for eight transmit antennas have comprised various unused symbol time slots $(50 \%$ of symbol time slots are unused in the code mentioned in [1]), where no useful information is transmitted. The number of unused symbol time slots in STBCs should be limited since, during those slots, the transmit antennas must be turned off. This is inconvenient from technical point of views, especially for the systems transmitting a hight data rate, i.e., the symbol time period is very small. In [2], two new orthogonal STBCs based on the AOD theory [3] were proposed for eight transmit antennas. In the proposed codes, namely $C_{1}$ and $C_{2}$, the number of unused symbol periods is only $25 \%$ and $12.5 \%$, respectively (see next section), compared to $50 \%$ in the conventional designs mentioned in literature, such as [1]. In addition, limiting the number of unused symbol time slots results in providing more space and symbol time slots for transmitting bits, i.e., providing more spatial and temporal diversity for those bits, and consequently, providing better bit error performance than the conventional code. This is clearly shown in the simulation results presented later in this paper Moreover, the authors took advantage of the property that, some symbols in the proposed codes appear more often than the others, in order to increase the code rate by utilizing higher level modulation schemes with higher transmission power for those symbols appearing more times in the codes. In this paper, the authors provide further research on these codes. Particularly, we utilize an 8-ary Quadrature Amplitude Modulation (8 QAM) scheme to further improve the bit error performance of the proposed codes. In addition, we examine the optimal inter-symbol power allocation in our proposed codes with various modulation schemes. It is a simple task to generalize the principles of the MMSs increasing the rate of STBCs and the optimal power allocation in multi-modulated STBCs mentioned in this paper to apply for STBCs of other orders, and therefore, we do not carry out this task here.

The paper is organized as follows. In Section II, the two new STBCs proposed for eight transmit antennas are derived. In the next section, the MMSs increasing the rate of the proposed codes are examined. The optimal ratios of symbol power in different modulation schemes for $C_{1}$ and $C_{2}$ in flat Rayleigh fading channels are examined in Section IV. Simulation results are presented in Section $\mathrm{V}$ and the paper is concluded by Section VI. The formulas for symbol enror probability of M-ary PSK signals in flat Rayleigh fading channels are derived in the Appendix.

\section{Two NEW ORTHOGONAL STBCS FOR EIGHT TRANSMIT ANTENNAS}

Orthogonal STBCs that can be used with complex signal constellations can be constructed by using CODs defined as follows:

Definition 1: A complex orthogonal design (COD) $\mathrm{X}$ of order $\mathrm{n}$ is an $n \times n$ matrix on the complex indeterminates $s_{1}, \ldots, s_{t}$, with entries chosen from $0, \pm s_{1}, \ldots, \pm s_{t}$, their complex conjugates $\pm s_{1}^{*}, \ldots, \pm s_{t}^{*}$, or their product with $i=\sqrt{-1}$, such that:

$$
X^{H} X=\left[\sum_{k=1}^{t}\left|s_{k}\right|^{2}\right] I_{n}
$$

where $X^{H}$ denotes the Hermitian transposition of $\mathrm{X}$ and $I_{n}$ is the identity matrix of order $n$.

CODs are strongly connected to the AODs [3]. The detailed theory of AODs, including limitations on the number of different variables for a given design order can be found in [4]. Drawing from the presented theory of the existence of AODs, we found two new CODs of order 8 , namely $C_{1}$ and $C_{2}$ (see 


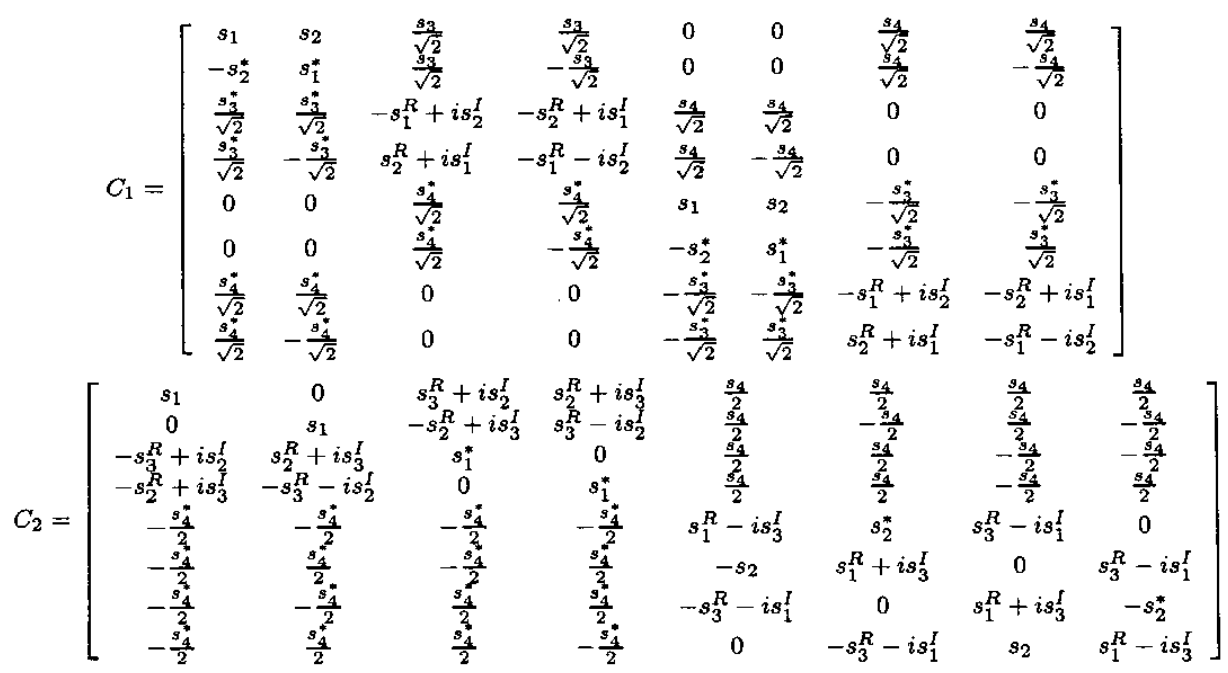

Fig. 1. Two new STBCs proposed for eight transmit antennas.

Figure 1), corresponding to the $\operatorname{AOD}(8 ; 1,1,2,2 ; 1,1,2,2)$ and $\operatorname{AOD}(8 ; 1,1,1,4 ; 1,1,1,4)$, respectively. It is easy to realize that, $C_{1}$ and $C_{2}$ satisfy the following equation:

$$
C_{i}^{H} C_{i}=\left[\sum_{j=1}^{4}\left|s_{j}\right|^{2}\right] I_{8} \quad i=1,2
$$

With single modulation, the proposed codes provide a code rate of $1 / 2$ as the conventional one.

\section{MMS TO INCREASE THE CODE RATE}

It is visible that, in $C_{1}$ and $C_{2}$, some symbols are transmitted in more than a single time slot per given antenna. In fact, in $C_{1}$, symbols $s_{3}$ and $s_{4}$ are transmitted twice as often as $s_{1}$ or $s_{2}$. In $C_{2}$, the symbol $s_{4}$ is transmitted four times as often as $s_{1}, s_{2}$ or $s_{3}$. Thus, by associating $s_{3}$ and $s_{4}$ in $C_{1}$ and $s_{4}$ in $C_{2}$ with symbols from multilevel complex modulation schemes and the remaining symbols in each of $C_{1}$ and $C_{2}$ with QPSK symbols, the overall code rates can be increased (there is, certainly, a tradeoff between the rate increase and the bit error performance). Particularly, if the MMSs (QPSK+8 PSK) and (QPSK+16 QAM) are used, then the code rate increases from $1 / 2$ to $5 / 8$ and $3 / 4$ for $C_{1}$, and to $9 / 16$ and $5 / 8$ for $C_{2}$, respectively [2]. The transmission power in each symbol time slot is equally allocated and normalized to 1 . It means that, $s_{3}$ and $s_{4}$ in $C_{1}$ are derived from a 2-power 8 PSK or 16-QAM signal constellation, while $s_{4}$ in $C_{2}$ is derived from a 4-power 8 PSK or 16-QAM signal constellation. All other symbols modulated by a QPSK signal constellation in the codes have unit power. Additionally, the MMS employing a QPSK signal constellation associated with an 8 QAM signal constellation (see Figure 2) can be utilized to further improve the bit error performance of the proposed codes $C_{1}$ and $C_{2}$ at the same bandwidth efficiency (same code rate) as when an 8 PSK signal constellation is used. Particularly, the symbols $s_{3}$ and $s_{4}$ in $C_{1}$ are modulated by a 2-power 8 QAM constellation, while $s_{4}$ in $C_{2}$ is modulated by a 4-power 8 QAM constellation. Other symbols in the codes are derived from a unitary QPSK constellation. The coordinates of the $8 \mathrm{QAM}$ signal points, presented as functions of the factor $A$, are given in the figure. It is easy to realize that, if the Euclidean distance between the

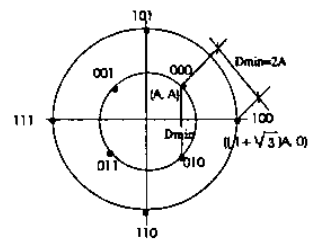

Fig. 2. 8 QAM signal constellation and bit mapping scheme.

two closest symbols in the constellation is $D_{\min }=2 A$ then the average transmitted signal power is $P_{a v}=4.73 A^{2}$ [5]. For the 2power and 4-power constellations, the value of $A$ in Figure 2 is 0.65 and 0.9196 , respectively. An 8 QAM signal constellation provides a better error property than an 8 PSK one, because, in order to have the same average power per symbol as in the former case, the Euclidean distance between the closest signal points in the later case $d_{\min }$ is smaller than that of the former case. Specifically, $d_{\min }=1.665 A$, i.e., $\frac{d_{\min }}{D_{\min }}=0.83$. Clearly, the orthogonality of the signals has been partially relaxed in the 8QAM constellation to increase the Euclidean distance between the closest signal points.

\section{OPTIMAL INTER-SYMBOL POWER ALLOCATION IN SINGLE MODULATION AND MMSS}

Allocating equally the power transmitted in each symbol period is optimal in terms of equal transmission among transmit antennas. However, in order to make sure whether the best error performance of the codes in different modulation schemes can be achieved, the dependence of the error probabilities of the proposed codes on the ratio between the power of the symbols in the codes must be examined. In this section, the symbol error rates ( $S E R \mathrm{~s}$ ) of QPSK single modulation, (QPSK+8PSK) and (QPSK+16 QAM) MMSs are examined. The $S E R$ s of QPSK, 8PSK and 16 QAM symbols in flat Rayleigh fading channels are (see (9), (11) in the Appendix at the end of this paper and (10.16), (10.35) and (10.42) in [6]):

$$
P_{Q P S K} \approx \frac{3}{4}\left[1-\frac{\mu}{\sqrt{2-\mu^{2}}}\right]
$$



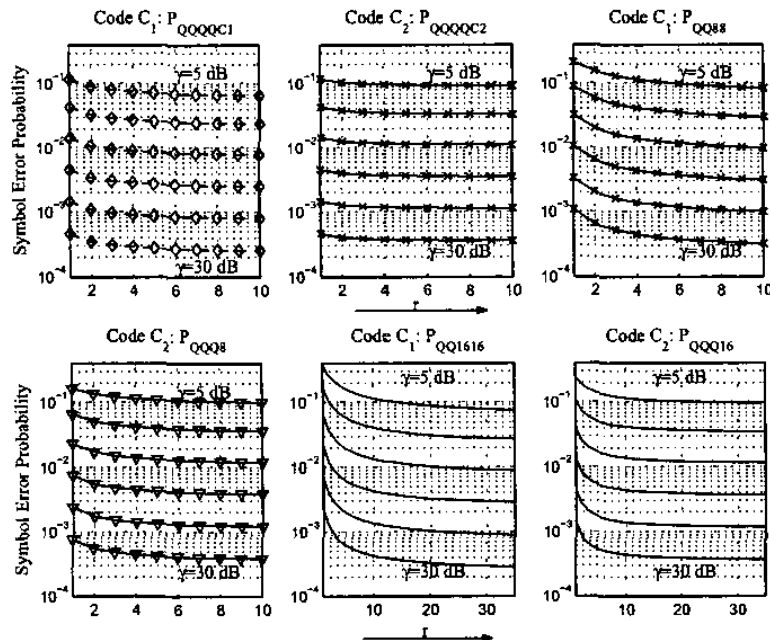

Fig. 3. $S E R$ v.s. $r$ in single modulation and MMSs depending on $\gamma$.

$$
\begin{aligned}
P_{8 P S K} & \approx \frac{7}{48 \gamma_{8} \sin ^{2} \frac{\pi}{8}} \\
P_{16 Q A M} & \approx \frac{1}{4 \gamma_{16}} \int_{0}^{\infty}\left[Q\left(\sqrt{\frac{y}{5}}\right)+Q\left(3 \sqrt{\frac{y}{5}}\right)\right] e^{\frac{-y}{4 \gamma_{16}}} d y \\
& +\left(1-\sqrt{\frac{2 \gamma_{16}}{2 \gamma_{16}+5}}\right)
\end{aligned}
$$

where $Q(x)=\frac{1}{\sqrt{2 \pi}} \int_{x}^{\infty} e^{-\frac{t^{2}}{2}} d t ; \mu=\sqrt{\frac{2 \gamma}{1+2 \gamma}} ; \gamma, \gamma_{8}$ and $\gamma_{16}$ are the signal-to-noise ratio (SNR) per bit of QPSK, 8 PSK and 16 QAM symbols, respectively.

Next, we calculate the $S E R \mathrm{~s}$ of $C_{1}$ and $C_{2}$ in different modulation schemes. Let us consider the case where the symbols $s_{1}$ and $s_{2}$ in the code $C_{1}$ are QPSK modulated, while $s_{3}$ and $s_{4}$ are 8 PSK modulated as an example. It is noted that, in each row (or column) in $C_{1}$, the power of the symbol $s_{j}(j=3,4)$ transmitted is $\left|s_{j}\right|^{2}$, i.e., only one symbol $s_{j}$ is transmitted, aithough, it may appear multiple times. Therefore, among four transmitted symbols, the probability when QPSK symbols are transmitted in $C_{1}$ is $50 \%$, and that when 8 PSK symbols are transmitted is $50 \%$, and consequently, the average $S E R$ of the code $C_{1}$ is:

$$
P_{Q Q 88}=\frac{1}{2} P_{Q P S K}+\frac{1}{2} P_{8 P S K}
$$

Let $E_{s_{k}}$ be the average power of the symbol $s_{k},(k=1 \ldots 4)$ and $r$ be the inter-symbol power ratio of the proposed codes, which is defined $r=\frac{E_{s_{i}}}{E_{s_{j}}}$, where $i=3,4 ; j=1,2$ for $C_{1}$ and $i=4 ; j=1, \ldots, 3$ for $C_{2}$. Clearly, in MMSs, $r$ is the ratio between the power of the higher level modulated symbols (8 PSK or 16-OAM) and that of the QPSK modulated ones. If we denote $N_{0}$ to be the variance of noise at the receive antenna, $\gamma_{s}$ the average SNR per symbol and $\gamma_{b}$ the average SNR per bit, then the power ratio can be rewritten as follows:

$$
r=\frac{E_{s_{i}} / N_{0}}{E_{s_{j}} / N_{0}}=\frac{\gamma_{s_{i}}}{\gamma_{s_{j}}}
$$

where $\gamma_{s}=\gamma_{b} \log _{2} M$ for an M-ary modulated symbol. Particularly, in the (QPSK+8PSK) MMS, $r=\frac{\gamma_{s_{i}}}{\gamma_{s_{j}}},(i=3,4 ; j=$

$$
\text { 1,2), or: } \quad r=\frac{3 \gamma_{8}}{2 \gamma}
$$

Therefore, if average symbol error probabilities are presented as functions of $r$ and $\gamma(S N R$ per bit of QPSK modulated symbols), then from (2), (3), (5) and (6), we have the average $S E R$ of $C_{1}$ as given below ${ }^{1}$

$$
P_{Q Q 88}=\frac{3}{8}\left(1-\sqrt{\frac{\gamma}{\gamma+1}}\right)+\frac{7}{64 r \gamma \sin ^{2} \frac{\pi}{8}}
$$

Similarly, the average $S E R$ for $C_{2}$ is:

$$
\begin{aligned}
P_{Q Q Q 8} & =\frac{3}{4} P_{Q P S K}+\frac{1}{4} P_{8 P S K} \\
& =\frac{9}{16}\left(1-\sqrt{\frac{\gamma}{\gamma+1}}\right)+\frac{7}{128 r \gamma \sin ^{2} \frac{\pi}{8}}
\end{aligned}
$$

Following this method to calculate symbol error probabilities, we derive the average $S E R$ s of QPSK single modulation and (QPSK+16QAM) MMSs in flat Rayleigh fading channels as:

- (QPSK+16QAM) multi-modulation:

$$
\begin{aligned}
P_{Q Q 1616} & =\frac{3}{8}\left(1-\sqrt{\frac{\gamma}{\gamma+1}}\right)+\frac{1}{2}\left(1-\sqrt{\frac{r \gamma}{r \gamma+5}}\right) \\
& +\frac{1}{4 r \gamma} \int_{0}^{\infty}\left[Q\left(\sqrt{\frac{y}{5}}\right)+Q\left(3 \sqrt{\frac{y}{5}}\right)\right] e^{\frac{-y}{2 r \gamma}} d y \\
P_{Q Q Q 16} & =\frac{9}{16}\left(1-\sqrt{\frac{\gamma}{\gamma+1}}\right)+\frac{1}{4}\left(1-\sqrt{\frac{r \gamma}{r \gamma+5}}\right) \\
& +\frac{1}{8 r \gamma} \int_{0}^{\infty}\left[Q\left(\sqrt{\frac{y}{5}}\right)+Q\left(3 \sqrt{\frac{y}{5}}\right)\right] e^{\frac{-y}{2 r \gamma}} d y
\end{aligned}
$$

- QPSK single modulation:

$$
\begin{aligned}
& P_{Q Q Q Q C 1}=\frac{3}{8}\left(1-\sqrt{\frac{\gamma}{\gamma+1}}\right)+\frac{3}{8}\left(1-\sqrt{\frac{r \gamma}{r \gamma+1}}\right) \\
& P_{Q Q Q Q C 2}=\frac{9}{16}\left(1-\sqrt{\frac{\gamma}{\gamma+1}}\right)+\frac{3}{16}\left(1-\sqrt{\frac{r \gamma}{r \gamma+1}}\right)
\end{aligned}
$$

Figure 3 presents the theoretical relation between $S E R$ s and $r$ depending on $\gamma$ for the above modulation schemes. In this figure, $\gamma$ runs from $5 \mathrm{~dB}$ to $30 \mathrm{~dB}$ with the pace of $5 \mathrm{~dB}$. We can realize that, when $r$ is small, then the higher $r$ is, the better the performance is. However, when $r$ increases, the curves become flat gradually. The value at which the curves become flat is the (smallest) optimal power allocation ratio $r_{\text {opt }}$. The optimal inter-symbol power ratios $r_{\text {opt }}$ for $C_{1}$ and $C_{2}$ in different modulation schemes are presented in Tables I. It is clear that the best symbol error performance can be achieved by the code $C_{2}$ in the QPSK single modulation and (QPSK+8 PSK) MMS, since, the power ratio of this code is $r=4$ while the (smallest) optimal power ratios are $r_{\text {opt }}=3$ and 4 , respectively. For the remaining modulation schemes, $r<r_{\text {opt }}$ and, consequently, there exists the gap between the

${ }^{\mathrm{I}}$ The channel $S N R$, which is used to simulate in this paper and is defined in Section $\mathrm{V}$ as $S N R=\frac{\sum_{k=1}^{4} E_{s_{k}}}{N}$, is a linear function of $\gamma$ (for a given value $r$ ). Additionally, the symbol error probability is a monotonically decreasing fuinction with respect to (w.r.t) $\gamma$ for a given value $r$ (see (7) for instance) function with respect to (w.r.t.) $\gamma$ for a given value $r$ (see $(7)$ for instance).
Hence, if the best error performance w.r.t. $\gamma$ is achieved, then that w.r.t. $S N R$ Hence, if the best error performance w.r.t. $\gamma$ is achieved, then that w.r.t. $S N R$
is also achieved. Based on these notes, in the paper, the authors search for the is also achieved. Based on these notes, in the paper, the authors search for the
optimal inter-symbol power ratio $r_{\text {opt }}$ w.r.t. $\gamma$, i.e., we search for the optimal power of the higher level modulated symbols corresponding to a given power of the QPSK modulated symbols. 
TABLE I

THE OPTIMALITY OF POWER ALLOCATION IN SINGLE MODULATION AND MMSs

\begin{tabular}{|l|c|c|}
\hline Modulation scheme & \multicolumn{2}{|c|}{ Min. $r_{\text {opt }}$} \\
\cline { 2 - 3 } & $C_{1}$ & $C_{2}$ \\
\hline Single QPSK & 6 & 3 \\
\hline QPSK + 8 PSK & 8 & 4 \\
\hline QPSK + 16 QAM & 25 & 20 \\
\hline
\end{tabular}

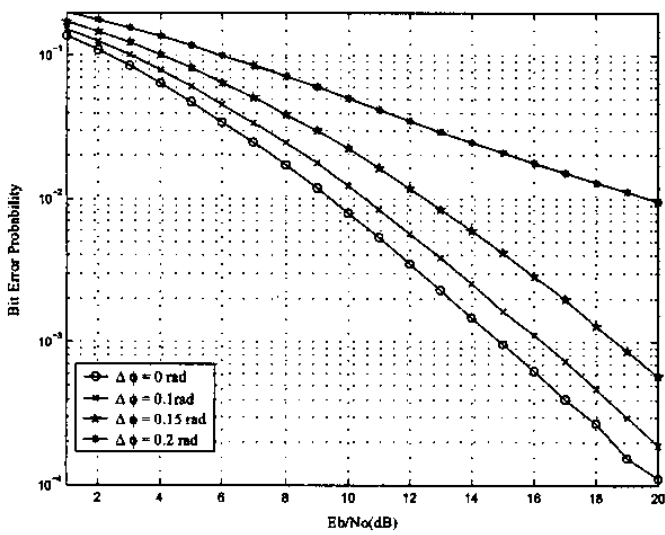

Fig. 4. $S E R$ v.s. $\gamma$ with the inter-symbol power ratio $r=2$ for $C_{1}, r=4$ for $C_{2}$ and with the optimal values $r_{o p t}$

error performance curves corresponding to $r$ and $r_{o p t}$, which is presented in Figure 4. From that figure, we realize that, the potential improvement for the code $C_{1}$ is $1 \mathrm{~dB}$ in both QPSK single modulation scheme and (QPSK+8PSK) MMS The potential improvements for $C_{1}$ and $C_{2}$ in the (QPSK +16 QAM) MMS are $6 \mathrm{~dB}$ and $1.75 \mathrm{~dB}$, respectively. The potential improvement is evaluated at $S E R=10^{-2}$. The potential improvement indicates that the error performance of the proposed codes, specially for $C_{1}$ in the (QPSK+16 QAM) MMS, can be much more improved by selecting $r$ closed to $r_{\text {opt }}$ with the penalty of unbalanced power transmission per symbol time slot at a given transmit antenna. In addition, it is observed from Figure 3 that, in the same MMS, the code $C_{1}$ may provide a higher code rate with a lower error probability than $C_{2}$ for large $r(r \geq 6$ in (QPSK+8PSK) MMS and $r \geq 15$ in (QPSK+16QAM) MMS) at any $\gamma$ in the considered range (5-30 dB). Hence, it is preferable to select $C_{1}$ if $r$ is large enough, provided that the balanced power transmission is not the necessary requirement of the system.

\section{Simulation Results}

In this section, the bit error properties of the codes $C_{1}$ and $C_{2}$ in single modulation as well as MMSs are presented. A system comprising eight transmit antennas and one receive antenna is considered. $S N R$ here means the channel $S N R$, i.e., the ratio between the total power of the received signals and the power of noise during each symbol time slot. Channels are assumed to be flat Rayleigh fading ones. The transmission gains and noise are assumed to be independent complex Gaussian random variables. In all simulations, the power of the signal transmitted in each symbol time slot in $C_{1}$ and $C_{2}$ is normalized to one. Figure 5(a) indicates that, at bit error rate $B E R=10^{-3}, C_{1}$ provides $0.4 \mathrm{~dB}$ bit error performance better than $C_{2}$, and $0.65 \mathrm{~dB}$ better than the conventional code [1], when QPSK single modulation is considered. This is intuitively interpreted as follows. Code $C_{1}$ provides more diversity (temporal and spatial) for four bits embedded in the symbols $s_{3}$ and $s_{4}$, while $C_{2}$ provides more diversity for only two bits in $s_{4}$. In other words, $C_{1}$ has a higher resistance to burst errors than $C_{2}$. Therefore, it is preferable to select $C_{1}$ for the case when QPSK single modulation is utilized for eight transmit antennas.

Figures 5(b) and 5(c) present the $B E R \mathrm{~s}$ of $C_{1}$ and $C_{2}$ in (QPSK+8PSK), (QPSK+8QAM) and (QPSK+16 QAM) MMSs. As mentioned in Section III, for the same MMS, $C_{1}$ provides a higher code rate than $C_{2}$. The performance of the conventional code [1] with those MMSs is presented here as the reference to evaluate the superiority of our codes (evaluation must be carried out in the same MMS, i.e., at the same bandwidth efficiency). It is noted that, for the conventional code, both symbols $s_{3}$ and $s_{4}$ are 8PSK or 16 QAM modulated in Figure 5(b), while only the symbol $s_{4}$ is 8PSK or 16 QAM modulated in Figure $5(\mathrm{c})$. The power transmitted per each symbol time slot is also normalized to one. Clearly, the MMS using an 8 QAM signal constellation provides better bit error performance than other schemes. Particularly, for the proposed codes, the $S N R$ gains achieved by the (QPSK+8 QAM) MMS are $0.15 \mathrm{~dB}$ for $C_{2}$, and 0.8 $\mathrm{dB}$ for $C_{1}$, respectively, to have the same $B E R=10^{-4}$ as in the (QPSK+8PSK) MMS. Additionally, at the same code rate, the proposed codes provide better bit error performance than the conventional code by around $1 \mathrm{~dB}$ in (QPSK+8PSK) MMS and $1 \mathrm{~dB}$ in (QPSK+16 QAM) MMS for case of $C_{1}$, and around $1.5 \mathrm{~dB}$ in (QPSK+8PSK) MMS and 2.7 $\mathrm{dB}$ in (QPSK+16 QAM) MMS for case of $C_{2}$, respectively, at $B E R=10^{-4}$. Therefore, at $B E R=10^{-4}$, the $S N R$ gains achieved by the (QPSK+8QAM) MMS are $1.65 \mathrm{~dB}$ for $C_{2}$ and $1.8 \mathrm{~dB}$ for $C_{1}$, compared to the conventional code with the (QPSK+8PSK) MMS.

\section{CONCLUSION}

In this paper, the MMSs are examined to increase the rate of our proposed codes for eight transmit antennas. In addition, the authors derive the optimal inter-symbol power ratios for the proposed codes in various modulation schemes. Based on the above consideration, the following conclusions can be derived. Firstly, when QPSK single modulation is utilized, it is recommended to select the code $C_{1}$ for eight transmit antennas as it provides the best $B E R$. Secondly, the (QPSK +8 QAM) MMS can be used to improve the performance of the codes proposed for eight transmit antennas, especially for $C_{1}$. Thirdly, it turns out that selecting the power ratio $r=4$ for $C_{2}$ is not only optimal in terms of equal power transmission per each symbol time slot, but also optimal in terms of achieving the best symbol error property in the QPSK single modulation and in the (QPSK+8 PSK) MMS in flat Rayleigh fading channels. Fourthly, the performance of the proposed codes can be remarkably improved, especially for the code $C_{1}$ in (QPSK+16 QAM) MMS, if the power ratio $r$ is selected closed to the optimal ratio $r_{o p t}$, with the penalty of unbalanced power transmission per symbol time slot at a given transmit antenna. Fifthly, in the same MMS, the code $C_{1}$ may provide a higher code rate with a better error performance than $C_{2}$ if the intersymbol power ratio is large enough, provided that the balance power transmission per each symbol time slot is not necessary 


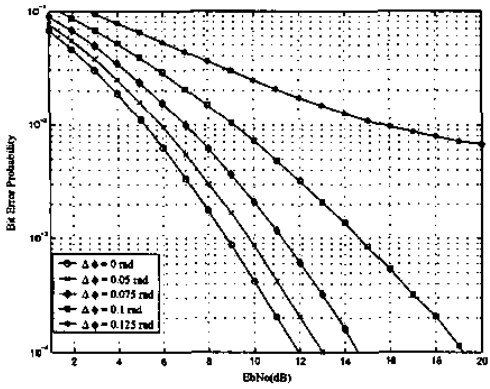

(a)

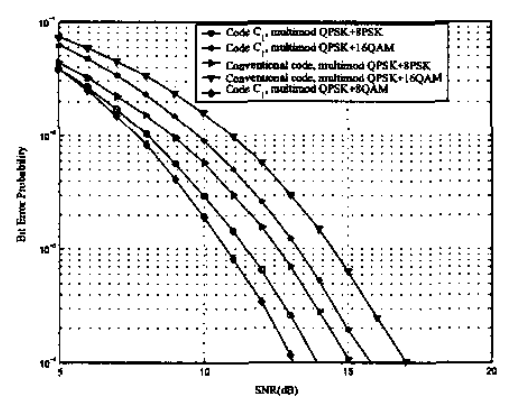

(b)

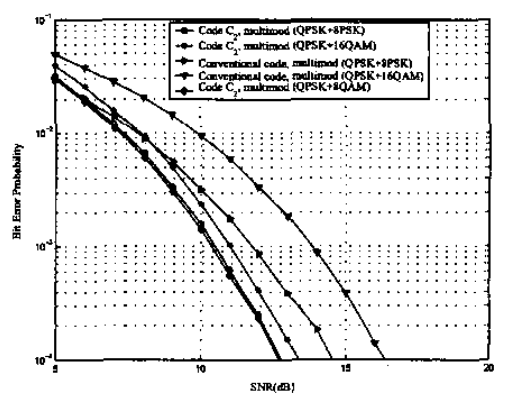

(c)

Fig. 5. (a): Comparison between the proposed codes and the conventional one in [1]; (b) and (c): Bit error performance of the code $C_{1}$ and $C_{2}$ with different MMSs.

property of the system. Finally, the principles examining the MMSs in order to increase the rate of STBCs and the optimal power allocation for multi-modulated STBCs mentioned in this paper can be generalized for STBCs of other orders, such as for the 4-ordered STBC in [1][7], without any difficulty.

\section{APPENDIX}

In this section, we derive the approximated symbol error probability of M-ary PSK signals in flat Rayleigh fading channels when $S N R$ per symbol is large enough. The symbol error probability of M-ary PSK signals in $L$-path Rayleigh fading channels is given below (see equation (14.4-38) in [5]):

$$
\begin{aligned}
P_{M} & =\frac{(-1)^{L-1}\left(1-\mu^{2}\right)^{L}}{\pi(L-1) !} \times \frac{\partial^{L-1}}{\partial b^{L-1}}\left\{\frac { 1 } { 1 - \mu ^ { 2 } } \left[\frac{\pi(M-1)}{M}\right.\right. \\
& \left.\left.-\frac{\mu \sin \left(\frac{\pi}{M}\right)}{\sqrt{1-\mu^{2} \cos ^{2}\left(\frac{\pi}{M}\right)}} \cot ^{-1}\left(\frac{-\mu \cos \left(\frac{\pi}{M}\right)}{\sqrt{1-\mu^{2} \cos ^{2}\left(\frac{\pi}{M}\right)}}\right)\right]\right\}
\end{aligned}
$$

where, by definition:

$$
\mu=\sqrt{\frac{\overline{\gamma_{c}}}{\bar{\gamma}_{c}+1}}=\sqrt{\frac{\left(\overline{\gamma_{b}} \log _{2} M\right) / L}{\left(\overline{\gamma_{b}} \log _{2} M\right) / L+1}}
$$

and $\overline{\gamma_{c}}$ and $\overline{\gamma_{b}}$ are the average SNR per channel and per bit, respectively. In flat Rayleigh fading scenario, we have $L=1$. Note that $\cot ^{-1}[\cot (-\phi)]=\pi-\cot ^{-1}(\phi),(0 \leq \phi \leq \pi)$, then we have:

$$
\begin{aligned}
P_{M} & =\frac{\left(1-\mu^{2}\right)}{\pi}\left\{\frac { 1 } { 1 - \mu ^ { 2 } } \left[\frac{\pi(M-1)}{M}-\frac{\pi \mu \sin \left(\frac{\pi}{M}\right)}{\sqrt{1-\mu^{2} \cos ^{2}\left(\frac{\pi}{M}\right)}}\right.\right. \\
& \left.\left.+\frac{\mu \sin \left(\frac{\pi}{M}\right)}{\sqrt{1-\mu^{2} \cos ^{2}\left(\frac{\pi}{M}\right)}} \cot ^{-1}\left(\frac{\mu \cos \left(\frac{\pi}{M}\right)}{\sqrt{1-\mu^{2} \cos ^{2}\left(\frac{\pi}{M}\right)}}\right)\right]\right\}
\end{aligned}
$$

When the $S N R$ per symbol satisfies: $\bar{\gamma}_{c} \gg 1$, such as $\bar{\gamma}_{c} \geq 10$ (i.e., $10 \mathrm{~dB}$ ), then $\mu \approx 1$. Therefore, we have:

$$
\begin{aligned}
P_{M} & \approx \frac{\left(1-\mu^{2}\right)}{\pi}\left\{\frac { 1 } { 1 - \mu ^ { 2 } } \left[\frac{\pi(M-1)}{M}-\frac{\pi \mu \sin \left(\frac{\pi}{M}\right)}{\sqrt{1-\mu^{2} \cos ^{2}\left(\frac{\pi}{M}\right)}}\right.\right. \\
& \left.\left.+\frac{\mu \sin \left(\frac{\pi}{M}\right)}{\sqrt{1-\mu^{2} \cos ^{2}\left(\frac{\pi}{M}\right)}} \cot ^{-1}\left(\cot \left(\frac{\pi}{M}\right)\right)\right]\right\} \\
& =\frac{(M-1)\left(1-\mu^{2}\right)}{M}\left\{\frac{1}{1-\mu^{2}}\left[1-\frac{\mu \sin \left(\frac{\pi}{M}\right)}{\sqrt{1-\mu^{2} \cos ^{2}\left(\frac{\pi}{M}\right)}}\right]\right\} \\
& =\frac{(M-1)\left(1-\mu^{2}\right)}{M}\left[\frac{\sqrt{1-\mu^{2} \cos ^{2}\left(\frac{\pi}{M}\right)}-\mu \sin \left(\frac{\pi}{M}\right)}{\left(1-\mu^{2}\right) \sqrt{1-\mu^{2} \cos ^{2}\left(\frac{\pi}{M}\right)}}\right]
\end{aligned}
$$

We can further simplify the above equation by noting that:

$$
\begin{aligned}
1-\mu^{2} & =\left[\sqrt{1-\mu^{2} \cos ^{2}\left(\frac{\pi}{M}\right)}-\mu \sin \left(\frac{\pi}{M}\right)\right] \\
& \times\left[\sqrt{1-\mu^{2} \cos ^{2}\left(\frac{\pi}{M}\right)}+\mu \sin \left(\frac{\pi}{M}\right)\right]
\end{aligned}
$$

Hence, (10) becomes:

$$
\begin{aligned}
P_{M} & \approx \frac{(M-1)\left(1-\mu^{2}\right)}{M} \frac{1}{\left[\sqrt{1-\mu^{2} \cos ^{2}\left(\frac{\pi}{M}\right)}+\mu \sin \left(\frac{\pi}{M}\right)\right]} \\
& \times \frac{1}{\sqrt{1-\mu^{2} \cos ^{2}\left(\frac{\pi}{M}\right)}} \\
& \approx \frac{(M-1)\left(1-\mu^{2}\right)}{M} \frac{1}{\left[2 \mu \sin \left(\frac{\pi}{M}\right)\right] \mu \sin \left(\frac{\pi}{M}\right)} \\
& =\frac{(M-1)\left(1-\mu^{2}\right)}{2 M \mu^{2} \sin ^{2}\left(\frac{\pi}{M}\right)}=\frac{(M-1)}{2 M \bar{\gamma}_{c} \sin ^{2}\left(\frac{\pi}{M}\right)}
\end{aligned}
$$

Therefore, we have:

$$
P_{M} \approx \frac{(M-1)}{2 M\left(\log _{2} M\right) \overline{\gamma_{b}} \sin ^{2}\left(\frac{\pi}{M}\right)}
$$

[1] O. Tirkkonen and A. Hottinen, "Square-matrix embeddable space-time block codes for complex signal constellations," IEEE Trans. Inform. Theory, vol. 48, no. 2, pp. 384-395, Feb. 2002.

[2] L. C. Tran, J. Seberry, B. J. Wysocki, T. A. Wysocki, T. Xia and Y. Zhao,"Two new complex orthogonal space-time codes for 8 transmit antennas," Electronics Lett., vol. 40, num. 1, pp. 55-56, Jan. 2004.

[3] A. V. Geramita and J. Seberry, Orthogonal designs: quadratic forms and Hadamard matrices, Lecture notes in pure and applied mathematics, vol. 43. Marcel Dekker, New York and Basel, 1979.

[4] G. Ganesan and P. Stoica, "Space-time block codes: a maximum SNR approach," IEEE Trans. Inform. Theory, vol 47, no. 4, pp. 1650-1656, May 2001.

[5] J. G. Proakis, Digital Communications, Fourth Edition, McGraw-Hill, 2001.

[6] W. T. Webb and L. Hanzo, Modern quadrature amplitude modulation - principles and applications for fixed and wireless communications, London: Pentech Press Limited, 1994.

[7] Vahid Tarokh, Hamid Jafarkhani and A. R. Calderbank, "Space-time blocks codes from orthogonal designs," IEEE Trans. Inform. Theory, vol. 45, no. 5, July 1999. 